\title{
LARGE TELESCOPE WIND LOADING
}

Fred F. Forbes

Advanced Development Program, National Optical Astronomy Observatories*

One important large telescope design study area is the dynamic image degradation caused by wind forces upon various telescope structures and optical elements. The need to establish rapid thermal equilibration with the observatory environment further complicates the wind exposure problem. on the other hand, a telescope deeply embedded in a large enclosure is subject to dome induced seeing difficulties unless great care is used to eliminate any thermal differences among telescope structure, optics, dome interior and inside and outside air. While dome seeing is outside the scope of this paper, it should not be neglected in the consideration of enclosure design because wind protection and seeing are inexorably coupled.

In their initial report on "Wind Loading of Large Astronomical Telescopes," Forbes and Gaborl (hereafter referred to as "Ref. 1"), have described wind power spectra obtained at Mt. Graham and Mt. Hopkins in Arizona and Mauna Kea in Hawaif. In addition to site wind data, gust power spectra were recorded for a variety of telescope orientations for the MMT (Multiple Mirror Telescope) at Mt. Hopkins and the UKIRT (United Kingdom Infrared Telescope) at Mauna Kea. In Ref. 1, Figure 8 and 10 are power spectral density plots for the MMT and UKIRT respectively. These gust spectra compare the measured site wind to that measured at the telescope mirror plane with the MMT at $45^{\circ}$ and the UKIRT zenith pointing. In Figure 9 of Ref. 1, pressure contour plots are given for the MMT at $45^{\circ}$ and zenith elevation. Further angular spectral data is presented in this paper for the MMT oriented in a variety of positions with respect to the site wind direction. Although the MMT and the CFHT have different enclosure and mount designs, general agreement is shown in this paper between the measured MMT wind loading and wind tunnel studies performed on a model of the CFHT at the Laboratoire d'Aerodynamique of the Ecole Nationale superieure D'Arts and Metiers in Paris, France by R. Grignon, M. Touratier and J. Driviere ${ }^{2}$ in 1973.

Although cup anemometer wind readings provide the standard wind reference, they do not adequately define the characteristics of the wind necessary in studies of structural wind loading. Specifically, simiu and $\operatorname{scanlan}^{3}$ give

\footnotetext{
* Operated by the Association of Universities for Research in Astronomy, Inc., under contract with the National science Foundation.
}

Proceedings of the IAU Colloquium No. 79: "Very Large Telescopes, their Instrumentation and Programs", Garching, April 9-12, 1984. 
the mean square value of the fluctuating along-wind deflection as:

$$
\sigma_{x}^{2}(z)=\int_{0}^{\infty} s_{x}(z, n) d n
$$

where $z$ is the structure elevation and $s_{X}(z, n)$ is the spectral density of the longitudinal velocity fluctuations at elevation $z$. The quantity $s_{x}(z, n)$ depends upon knowledge of the wind spectra which is best provided by Pitot tube or hot wire anemometers as shown in Figures 7,8 and 9 of Ref. 1, and is given by Simiu and scanlan as:

$$
\frac{n S(z, n)}{u_{\star}^{2}}=\frac{200 f}{(1+50 f)^{5 / 3}}
$$

for which $u_{\star}$ is the frictional velocity discussed below as a site related parameter to be measured. While site spectra shown in Ref. 1 indicate a Kolmogorov $-5 / 3$ power law dependency, a considerable number of wind loading studies have been based upon the Davenport $-4 / 3$ power law ${ }^{3}$. The Kolmogorov spectra more accurately describes the measured wind spectra as reported by others $4,5,6,7$. Simiu and $\operatorname{scanlan}^{8}$ have shown the existance of a significant high frequency excess associated with the Davenport model, a fact of utmost concern to those attempting to project structure responses to high frequency wind contributions.

The quantity $\sigma_{x}{ }^{2}(z)$ is useful for telescope wind loading studies because it allows structure motion and image motion to be related by ${ }^{9}$ :

$$
\delta \theta=\frac{\delta \mathbf{x}}{f_{1}}\left[1-\frac{f_{1}}{f}\right]
$$

where $\delta \theta$ is the image motion due to rotation of the optical axis or due to secondary displacement relative to the optical axis, $\delta \mathbf{x}$ is secondary decenter relative to the optical axis, $f$ is the final focal length and $f_{1}$ is the primary focal length. Here $\delta x$ given in Ref. 9 by Nelson, and $\sigma_{x}(z)$ of Simiu and Scanlan are directly equatable at the secondary mirror location.

Large telescope wind loading is linked to the specific placement of the telescope in relation to the local orographic effects. Unfortunately, by attempting to avoid direct winds, the resultant seeing degradation due to wake turbulence can negate the gains afforded by increased aperture. Wind tunnel studies of wake turbulence for the Pic du Midilo and Mauna Keall sites dramatize the role that terrain and wind direction play upon site turbulence. In practice, site roughness or roughness length zo may be characterized by the observation of wind for a variety of wind directions, speeds and elevations and is related to the turbulent eddy size at the ground level. simiu and Scanlan 12 define the terrain roughness parameters $z_{0}$ and $z_{d}$ with the latter, 
the zero plane displacement related to the height of surrounding trees $\bar{h}$ by $z_{d}=0.75 \bar{h}$. Estimates of from .01 and 0.3 for the terrain roughness $z_{0}$ at Mauna Kea to that at Mt. Graham and Hopkins respectively allow the calculation of frictional velocity $u_{*}$ as:

$$
u_{*}=\frac{U\left(z_{R}\right)}{(2.5) \ln \left[\left(z_{R}-z_{d}\right) / z_{0}\right]}
$$

Where $z_{R}$ is the reference height, usually taken to be 10 meters in meteorological work. It is evident that $z_{0}$ may be determined for a site by knowing the average tree height $\bar{h}$ and the mean wind velocity $U\left(z_{R}\right)$ at the reference height $z_{R}$. For a treeless site, the frictional or shear velocity expression reduces to

$$
u_{*} 2 \frac{U\left(z_{R}\right)}{(2.5) \ln \left[\frac{z_{R}}{\left[z_{0}\right.}\right]}
$$

Using equations 2 and 5, and the measured spectra in Figures 8 and 10 of Ref. 1, the roughness parameter $z_{0}$ was calculated to be about one order of magnitude too large for both Mauna Kea and Mount Hopkins. Although the cup anemometer and pitot spectra are in agreement, the excess roughness remains unexplained unless equations 4 and 5 are unrepresentative of mountain terrain.

Telescope wind loading is dependent on enclosure configuration as well as wind velocity and direction. There are three general classes of telescope/enclosure arrangements: 1) embedded, 2) flushed and 3) exposed. The exposed telescope, while protected by a roll-off building when not in use, is fully subject to site wind loading during observational periods. The effects of wind loading have been studied for exposed solar collectors 13 and for a model of the CFHT ${ }^{14}$. In both studies, a strong wind torque peak is experienced at $90^{\circ}$ relative to the site wind direction as shown for the CFHT in the dashed "No Dome" curve of Figure 1. Here, telescope torque $C_{\alpha}$ given by Grignon, Touratier and Driviere as:

$$
\mathrm{c}_{\alpha}=\mathrm{c}_{\mathrm{m}_{\alpha}} \cdot \mathrm{v} \cdot \frac{\rho}{2} \cdot \mathrm{v}_{\mathrm{o}}^{2} \cdot \mathrm{E}^{-3}
$$

$$
\text { where } \begin{aligned}
\mathrm{c}_{\alpha} & =\text { wind induced torque } \\
c_{\mathrm{m}_{\alpha}} & =\text { torque coefficient } \\
\mathrm{V} & =\text { reference volume } \mathrm{V}=\mathrm{SL} \\
\rho & =\text { density of } \operatorname{air} \\
\mathrm{v}_{\mathrm{O}}^{2} & =\text { wind speed } \\
\mathrm{E} & =\text { scale factor }=\frac{1}{50}
\end{aligned}
$$


In the heliostat wind tunnel study of $\mathrm{J}$. Xerikos et a113, the arag force $C_{F_{X}}$ peak at $90^{\circ}$, shown in their Figure 8 , is similar to the No-Dome CFHT results indicating maximum torque experienced at right angles to the flow.

Site wind transfer into and thru an enclosure is described in the diagrams of Figure 2 for the embedded and flushed cases. Site wind throughput for embedded and flushed enclosure configurations, based on the data of Melaragno15, are summarized in part in Table 1. These in situ data indicate a relative insensitivity to wind angle for the embedded case and a strong forward angular dependency for the flushed arrangement.

In Table 1, throughput $x$ is defined as the percentage of wind transfered into or through the enclosure in the presence of a wind $\mathrm{V}$ referenced as $100 \%$.

TABLE I

Site wind Throughput for Embedded and Flushed Enclosure Configurations

\begin{tabular}{lc|ccc}
\hline $\begin{array}{l}\text { Site Wind } \\
\text { Angle, } \theta^{\circ}\end{array}$ & $\begin{array}{c}\text { Embedded } \\
\text { Throughput X8V }\end{array}$ & $\begin{array}{c}\text { Site Wind } \\
\text { Angle, } \theta^{\circ}\end{array}$ & $Y$ & $\begin{array}{c}\text { Flushed } \\
\text { Throughput X8V }\end{array}$ \\
\hline 0 & 4.7 & 0 & 1 & 32 \\
22.5 & 3.6 & 0 & 2 & 36 \\
45 & 3.3 & 0 & 3 & 47 \\
67.5 & 3.8 & 45 & 1 & 41 \\
135 & 3.6 & 45 & 2 & 62 \\
& & 35 & 65 \\
\hline
\end{tabular}

The wind tunnel results of Figure 1 are comparable to the Table 1 data for the embedded case related to the fully exposed telescope. In Figure 1 , about 38 of the "No Dome" torque is experienced by a telescope embedded in an enclosure with a square opening slightly larger than the primary mirror regardless of the opening orientation.

The wind tunnel model data compare favorably to actual in situ measurements taken at the MMT (Ref. 1, and Figures 3 and 4 , for the flushed configuration in Table 1 with $Y=1$, that is, a flushed enclosure with an exit port about $1 / 3$ of the input port linear dimension $L$ ). In addition, there exists a strong angular effect similar to that of the "Fully open Shutter" configuration of Figure 1. In both Figure 3 and 4 , the MMT was turned into the wind and the wind energy falling on the primary mirror plane was measured. In Figure 3, the telescope was brought from a nearly horizontal position to zenith pointing while the building was oriented into the wind. 


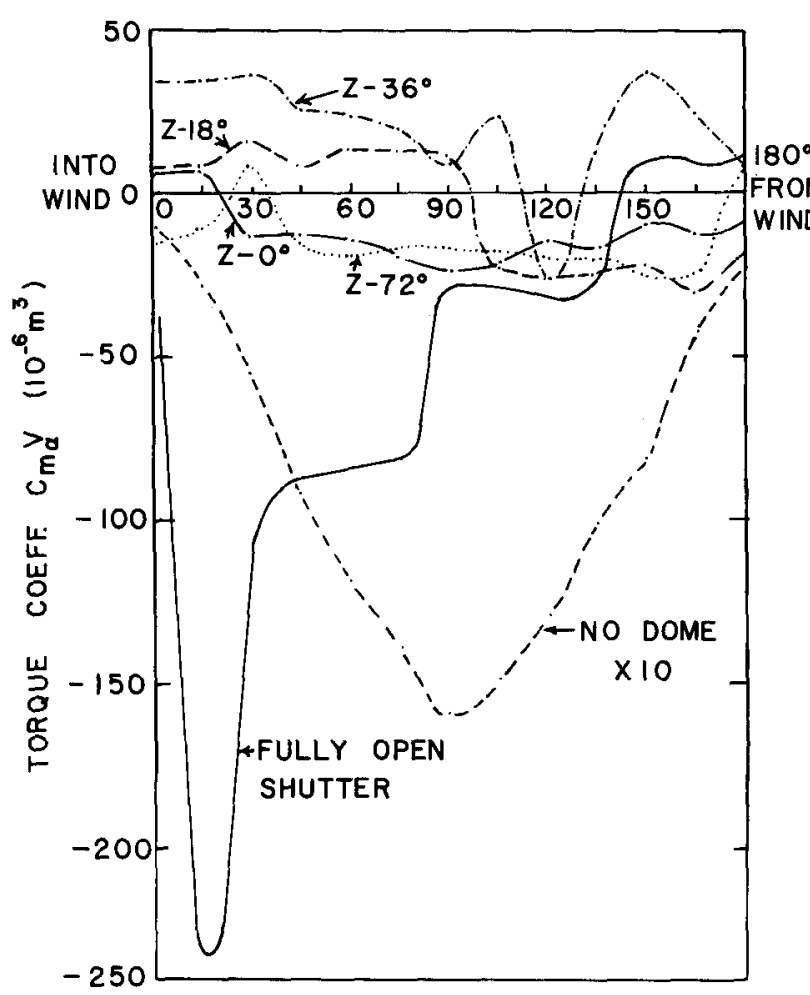

Figure 1

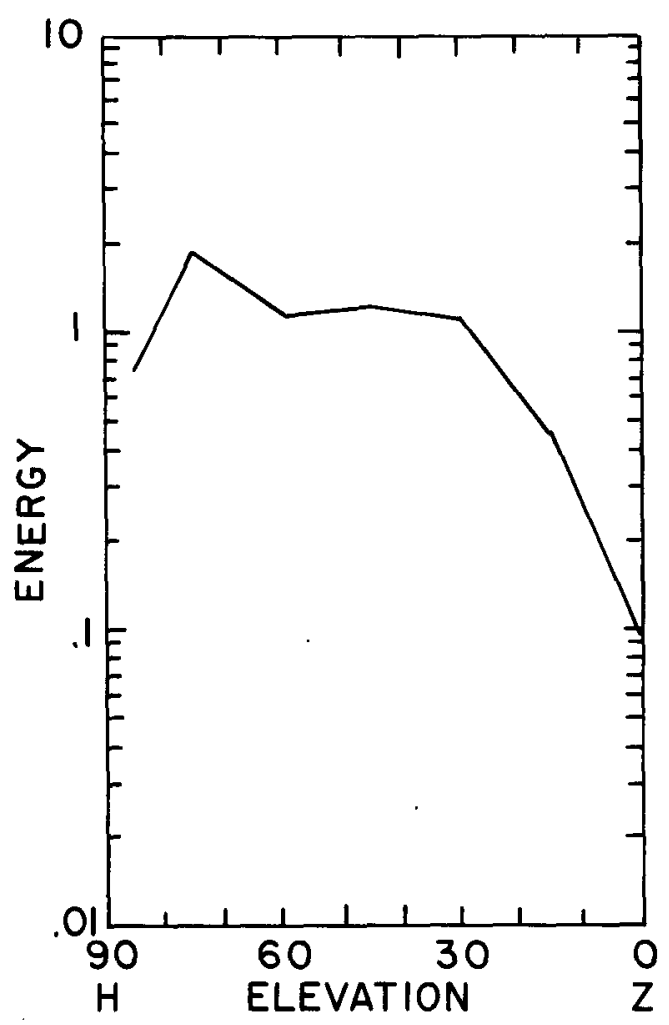

$100 \% \mathrm{~V} /$

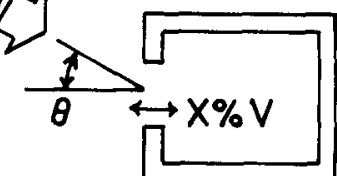

EMBEDDED

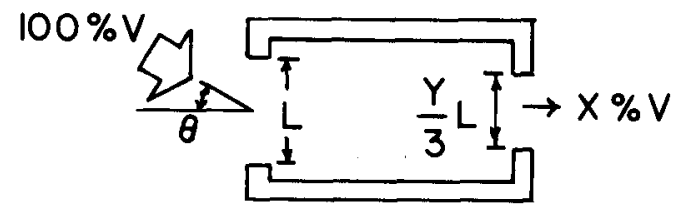

FLUSHED

Figure 2

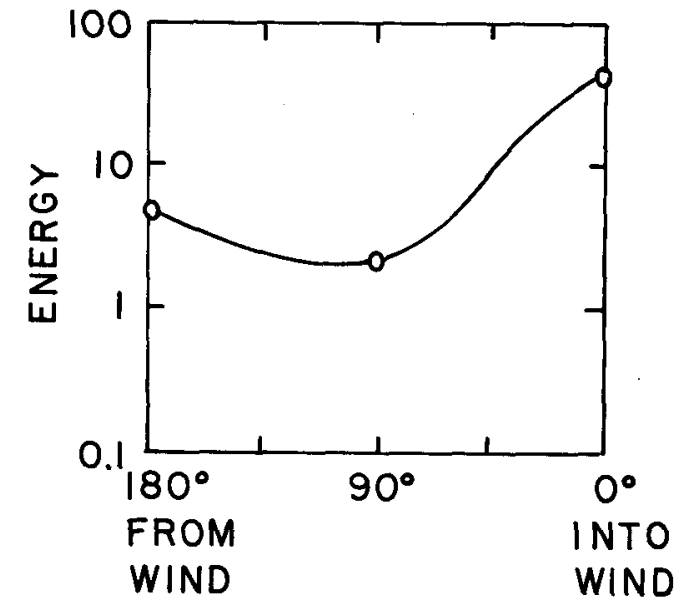

Figure 4

Figure 3 
The broad peak from $20^{\circ}$ to $60^{\circ}$ with respect to the horizontal wind direction in Figure 3 represents an intermediate situation between the "No Dome" and the "Fully Open Shutter" measurements in Figure 1. Moreover, the ratio of a factor of 20 over the $90^{\circ}$ range is in general agreement with the "Fully Open Shutter" curve of Figure 1 . In Figure 4, the effect of MMT building rotation is shown for which a factor of about 20 decrease in energy exists between the full into the wind and $90^{\circ}$ with-respect-to-the-wind building orientations. The increase of energy experienced when the MMT is $180^{\circ}$ out of the wind is perhaps due to spill over into the open shutter on the MMT roof.

Similarly, the wind energy spectral shift for the MMT was studied under various telescope and building orientations as measured at the MMT mirror plane. As shown in Figure 5, a significant fraction of energy is shifted from the low to the high frequencies with the telescope zenith pointing as compared to the orientations more directly into the horizontal wind. This effect may be due to a more efficient vortex generation when more of the telescope optical support structure is exposed at high pointing angles, or the effect could be due to a shutter filtering effect reducing the low frequency energy reaching the mirror plane. With the telescope set at $45^{\circ}$ elevation, the result of Figure 6 shows a nearly uniform decrease in energy of nearly 20 for the $90^{\circ}$ and out-of-the-wind case compared to the into the wind reference. These results are in agreement with both the wind tunnel measurements in Figure 1 and the in situ measures shown in Figures 3 and 4. The lack of low frequency attenuation in Figure 6 is perhaps related to the fact that the telescope is positioned at an angle sufficiently low to experience the direct wind spectra as in Figure 5 for the $30^{\circ}$ and $75^{\circ}$ telescope orientations. Further, the energy reduction seems to follow the expected reduction predicted by Melaragno in Figure 2 and Table 1.

In conclusion, the full scale and wind tunnel test results suggest some possible means of reducing wind loading effects on large telescopes. The site, enclosure, and telescope may all be modified to reduce deteriorous wind, however, the selection of a site with natural wind shielding will generally have an associated degraded seeing. Thus, by avoiding locations of full wind exposure at sites with known high winds, telescope wind loading is likewise reduced at the potential risk of increased turbulence. The selection of an enclosure which fully protects the telescope from wind loading likewise creates an observational environment subject to local seeing and associated image degradation. Woolf and Ulichl6 have addressed the problem 


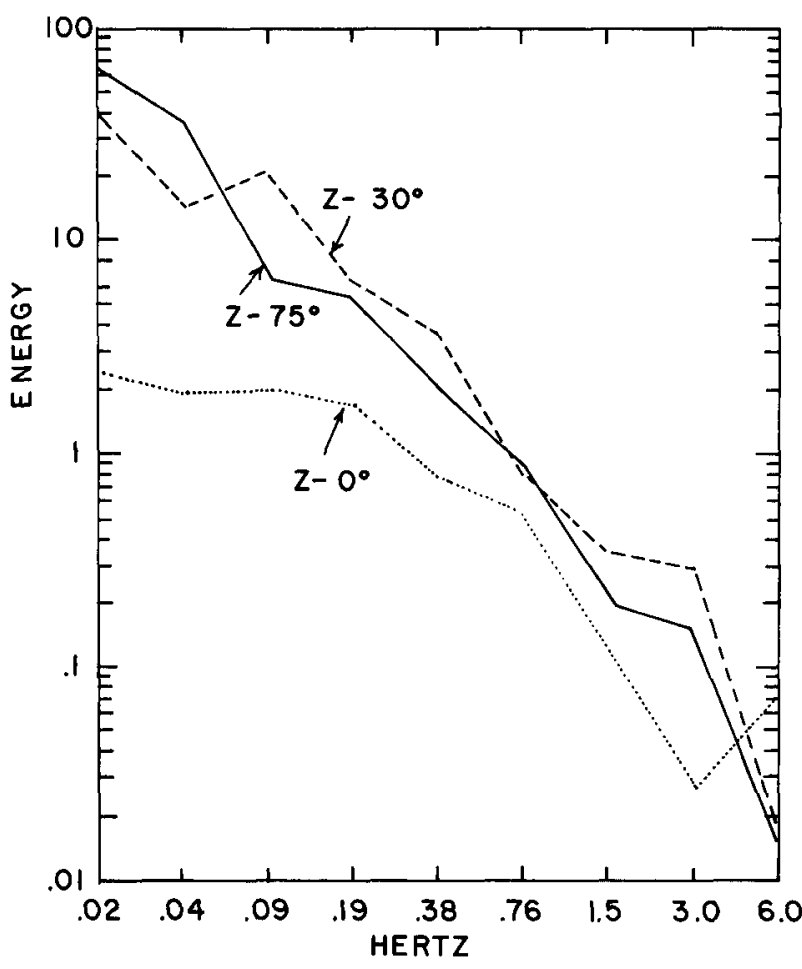

Figure 5

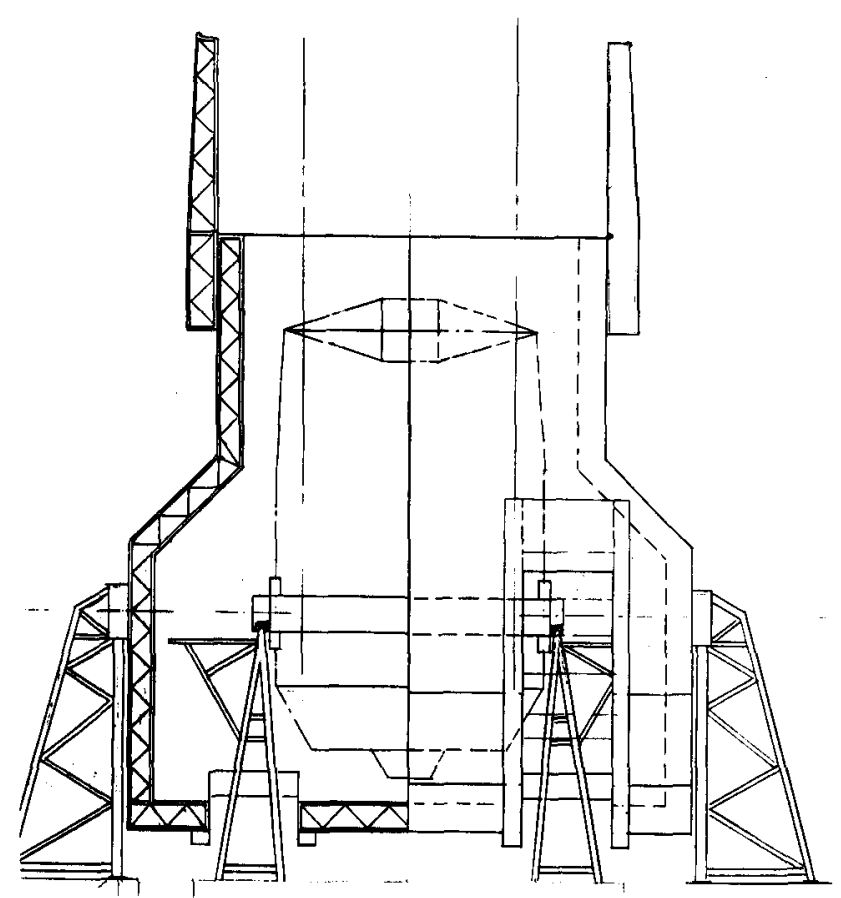

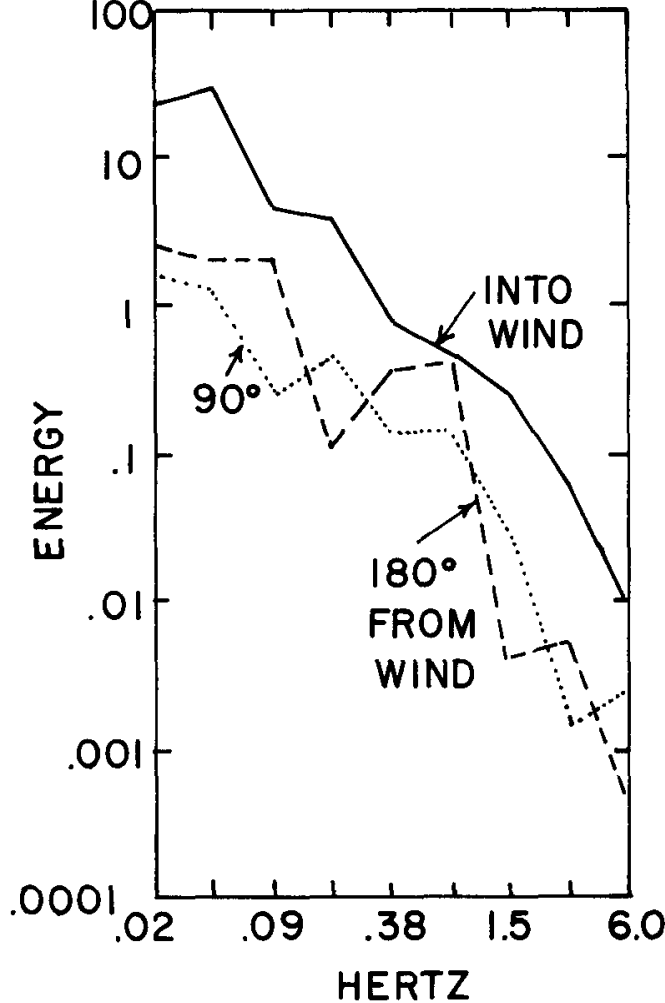

Figure 6

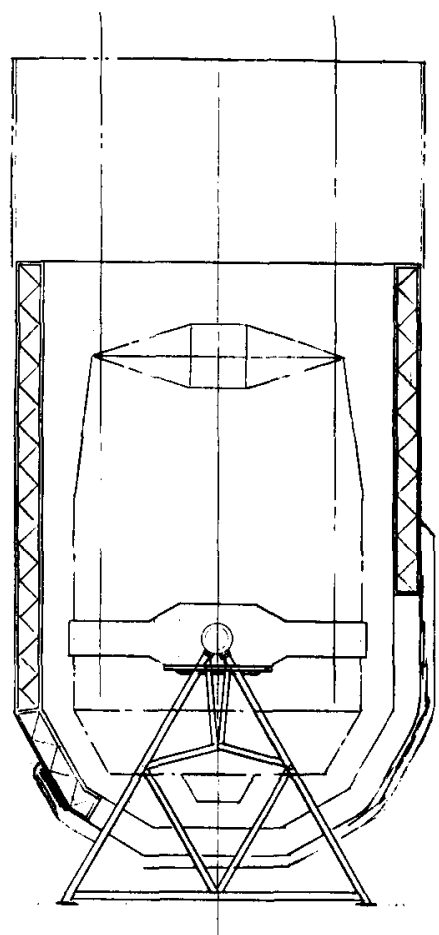

Figure 7 
of seeing and wind loading and conclude that a flushed enclosure similar to the MMT, designed to provide adequate wind protection, simplifies the local seeing problem due to the rapid equilibration of enclosure interior and ambient temperatures. However, while the embedded telescope resides in a more stable environment, active thermal control is required to insure identical inside/outside temperatures of air and surfaces. To the extent that gradients in temperature exist, dome seeing prevails, as has been shown by a number of authors17-21. One possible solution is shown in Figure 7, where a shroud envelopes the telescope optical support structure and effectively acts as a "dome" which coarsely tracks the telescope mount in altitude as well as azimuth. The shroud may extend well beyond the secondary optics thereby acting as an embedded telescope enclosure. Further, active temperature control would be extended to all shroud interior surfaces thereby providing the necessary close thermal tracking. The slits at the enclosure base which accommodate enclosure motion may be tightly sealed to allow the active cooling to be most effective or the slits could be more open to flush the instrument if so desired. The volume and cooling requirement reduction for the shroud compared to a conventional enclosure would provide a cost reduction.

Finally, wind screens and fences added to the enclosure can greatly reduce wind throughput to the telescope. Cermak and Peterka 22 have described vortex generators combined with triangular fences which absorb wind energy. Likewise, Murota 23 has shown that screens with soldarity ratios of 0.2 provide drag coefficients of 0.2 . J. Xerikos, et al, have concluded 24 that a wind fence can significantly reduce aerodynamic loads experienced by individual or clustered heliostats. By placing screens and fences far in front of the shroud enclosure aperture, shroud wind loading may be reduced due to screen porosity, and telescope wind loading is reduced because of screen protection. Seeing need not be affected if precautions are taken to avoid any temperature differential between screen and air. Gartshore, Khanna and Laccinole 25 have demonstrated the reduction in tube structure Reynolds number by addition of fins or strakes. A strake, or longitudinal fin, $1 / 5$ th of the cylinder diameter, can increase the wind velocity required for cylinder resonance by a factor of 2 and can reduce the peak Reynolds number by from 2 to 20 dependent on the degree of turbulence of the flow. 
By utilizing a combination of the wind loading reduction techniques, suggested above, large telescope image degradation due to wind shake may be reduced to acceptable limits without introducing deteriorous seeing effects.

\section{References}

1. Forbes, F., and Gabor, G., "Wind Loading of Large Astronomical Telescopes," UC TMT Report No. 83 and SPIE Proceedings, Vol. 332, "Conference on Advanced Technology Telescopes, Tucson, AZ, U.S.A., March 1982.

2. Grignon, R., Touratier, M. and Driviere, J., "Telescope Implante Aux Iles Hawai Rapport No. 2, Etude Aérodynamique Du Télescope," Ecole Nationale Superieure D'Arts Et Metiers, Paris, 1973 and "Aerodynamical Studies made for the CFHT Observatory," summary by Pierre $Y$. Bely, Canada-France-Hawaii Telescope Corporation, Kamuela, HI., U.S.A., 1983.

3. Simiu, E., and Scanlan, R. H., "Wind Effects on Structures," John Wiley, New York, U.S.A., 1978, p. 186.

4. Okubo, T., Narita, N., Yokoyama, K., and Sato, H., "Field Observations of Aerodynamic Behavior of Long-Span Bridges," Proceedings of the Fifth International Conference on Wind Engineering, Fort Collings, Co., U.S.A, July 1979, Edited by J. E. Cermak, Vol. 2, p. 830

5. Schnabel, W., and Plate, E. J., "Prototype and Model Experiments on Wind-Induced Vibrations of a Steel Tower," Proceedings of the Fifth International Conference on wind Engineering, Fort collins, Co., U.s.A., July 1979, edited by J. E. Cermak, Vol. 2, p. 762.

6. Shiraishi, Naruhito, "Aerodynamic Responses of Bridges to Natural Winds," Proceedings of the Second U.S.A. - Japan Research Seminar on Wind Effects on Structures, edited by H. Ishizaki, and Arthur N. L. Chiu, University of Hawaii Press, U.S.A., 1974.

7. Kawai, H., Katsura, J., and Ishizaki, H., "Characteristics of Pressure Fluctuations on the Windward wall of a Tall Building," Proceedings of the Fifth International Conference on wind Engineering, Fort Collins, CO., U.S.A., July 1979, edited by J. E. Cermak, Vol. 1, p. 522.

8. Simiu, E., and Scanlan, R. H., "Wind Effects on structures," John Wiley, New York, U.S.A., 1978, p. 56.

9. Nelson, Jerry, "The Effects of Wind Loads on the Ten Meter Telescope," UC TMT Report No. 47, February 1983. 
10. Pagés, J. P., Rosch, J., et Saissac, "Influence of Aerodynamical Wakes on the Quality of Astronomical Images at Pic-du Midi," Astronomy and Astrophysics, 28, 1973, p. 403-413.

11. Grignon, R., Touratier, M., and Driviere, J., "Telescope Implante Aux Iles Hawai Rapport No. 1, Etude Aérodynamique D'un Modéle Topographique," Ecole Nationale Superieure D'Arts et Metiers, Paris, Sept. 1972.

12. Simiu, E., and Scanlan, R. H., "Wind Effects on Structures," John Wiley, New York, U.S.A., 1978, p. 251-3.

13. Xerikos, J., Tang, H. H., Cermak, J. E., and Peterka, J. A., "The Aerodynamics of Heliostats for Solar Power Plant Applications," Proceedings of the Fifth International Conferences on wind Engineering, Fort Collins, Co., U.S.A., July 1979, Vol. 2., p. 1173-1183.

14. Grignon, R., Touratier, M., and Driviere, J., "Telescope Implante Aux Iles Hawai Rapport No. 2, Etude Aérodynamique Du Télescope," Ecole National Superieure D'Arts Et Metiers, Paris, 1973 and "Aerodynamical Studies Made for the CFHT Observatory," summary by Pierre Y. BelY, Canada-France-Hawaii Telescope Corporation, Kamuela, HI., U.S.A., 1983.

15. Melaragno, Michele, "Wind In Architectural and Environmental Design," Van Nostrand Reinhold Col, New York, U.S.A., 1982, p. 321-368

16. Woolf, N. J., and Ulich, B. L., "Gone with the wind or Sailing and seeing with a Giant Telescope," preprint of the steward Observatory, No. 497, the University of Arizona, Tucson, AZ., U.S.A., 1983

17. Woolf, N., "Dome Seeing," P.A.S.P., Vol. 91, p. 523-529, 1979.

18. Beckers, J. M., "MMT seeing Experiments in 1980," MMTO Technical Report No. 10, Tucson, AZ., U.S.A., 1980.

19. Gillingham, P. R., "Seeing Measurements at the Anglo-Australian Telescope," SPIE Proc., Advanced Technology Telescopes II, Iondon, Sept. 1983.

20. Forbes, F. F., "Dome Induced Image Motion," SPIE Proc., Vol. 332, Advanced Technology Telescopes, Tucson, AZ., U.S.A., March 1982.

21. Woolf, N. J. and Ulich, B. L., "Gone with the Wind, or Sailing and Seeing with a Giant Telescope," preprint of the Steward Observatory, No. 497, The University of Arizona, Tucson, AZ., U.S.A., 1983.

22. Cermak, J. E., and Peterka, "Wind Pressures on a House Roof," Proceedings of the Second U.S.A. - Japan Research Seminar on Wind Effects on Structures, edited by H. Ishizaki, and Arthur N. L. Chiu, University of Hawaii Press, 1974. 
23. Xerikos, J., Tang, H. H., Cermak, J. E., and Peterka, J. A., "The Aerodynamics of Heilostats for Solar Power Plant Applications, Proceedings of the Fifth International Conference on Wind Engineering, Fort Collins, Co., U.S.A., July 1979, edited by J. E. Cermak, Vol. 2, p. 1173-1783.

24. Gartshore, I. S., Khanna, J. And Laccinole, S., "The Effectiveness of Vortex spoilers on a Circular Cylinder in Smooth and Turbulent Flow," Proceedings of the Fifth International Conferences on Wind Engineering, Fort Collins, Co., U.S.A., July, 1979, Vol. 2 .

\section{DISCUSSION}

R. Wilson: You mentioned the possibilities of windbreak using "strakes" or fins compared with cylinders. For a pull-up windbreak, what proportion of effective closed surface and what geometrical form would you recommend?

F. Forbes: The windbreak should be about $1 / 3$ closed, $2 / 3$ open. The geometrical form is uncritical - whatever is technically convenient.

Note: Strakes and fins are useful additions to the cylindrical members of the telescope optical support structure. They reduce oscillation amplitude and increase the wind velocity necessary to cause resonance. A pull-up wind break using screens of solidity ratio of .01 to .20 would be an effective wind shield. Triangular wind fences of $25 \%$ solidity also would be effective providing temperatures are equilibriated. 